Neurosurg Focus 6 (4):Article 13, 1999

\title{
Improved safety of neuroendoscopic third ventriculostomy by using an operative Doppler ultrasound probe
}

\section{Technical note}

\section{Michael Vloeberghs, M.D., and Maria Cartmill, F.R.C.S.}

Department of Pediatric Neurosurgery, University of Nottingham, Nottingham, United Kingdom

In this paper the authors describe the use of a Doppler device, originally built for intravascular use, that is passed through a flexible neuroendoscope during a neuroendoscopic third ventriculostomy (NTV) procedure to treat for hydrocephalus. There was no morbidity associated with the use of this Doppler probe, and the procedure was not significantly lengthened. The Doppler probe was very accurate in locating the position of the basilar artery tip and assured the safety of NTV.

Key Words * neuroendoscopic third ventriculostomy * hydrocephalus * third ventricle anatomy * cerebral circulation * doppler velocity measurement

A new technical addition to the neuroendoscopic treatment of hydrocephalus is described. It is not the authors' intention to discuss either the method or the indications for neuroendoscopic third ventriculostomy (NTV) but to demonstrate the ease with which potential harm to the tip of the basilar artery (BA) or adjacent vessels can be avoided by using a Doppler ultasound probe.

\section{DESCRIPTION OF TECHNIQUE}

An 11 month-old male infant presented with gross hydrocephalus as the result of fourth ventricle obstruction by a large vermis tumor that proved suitable for NTV. After appropriate consent for NTV and the operative use of the Doppler probe was obtained, anesthesia was induced, and a "classic" right precoronal burr hole was made to gain access to the ventricular system. A flexible neuroendoscope loaded with a Doppler probe was introduced into the ventricular system in the customary way. The Doppler probe was first brought close to the tip of the BA and then over the area chosen for the ventriculostomy. Where the area behind the sella turcica was silent (Fig.1 upper), the signal over the tip of the BA was obvious (Fig. 1 lower). 


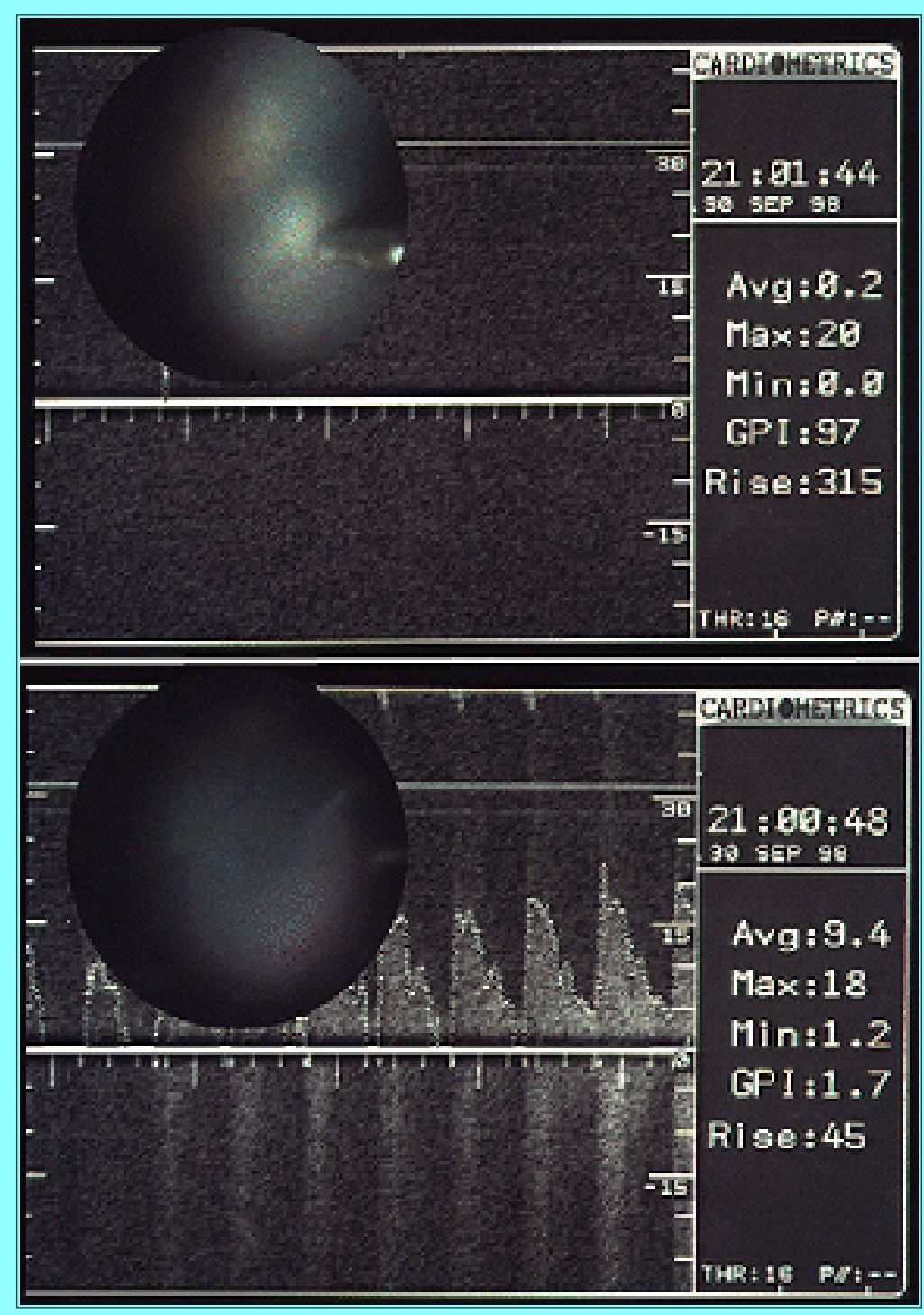

Fig. 1. Upper: The ultrasound (the background) and video of the procedure (the circle) are recorded simultaneously and reedited onto a screen in screen format. This figure shows the transparency of the sella turcica (top of the circle) and the Doppler probe (bottom right). The tip of the probe is pressed onto the floor of the third ventricle. The background screen shows there is no velocity signal below the third ventricle in that area, which corresponds to a depth of approximately $4 \mathrm{~mm}$. Lower: Here the Doppler probe is placed near the tip of the $\mathrm{BA}$, and a convincing velocity signal is produced. Maximum velocity in the BA is 18 $\mathrm{cm} / \mathrm{second}$.

This area remained silent even when the probe was pressed onto the floor of the third ventricle. The Doppler wire was then exchanged for a monopolar electrode, and the floor of the third ventricle was opened using cutting diathermy under constant irrigation with Hartman's solution. The NTV was uneventful.

The monopolar electrode was then exchanged for the Doppler probe, and the probe was brought back to the tip of the BA and the NTV. The area behind sella turcica remained silent, and upon inspection of the surgical site no vessels were seen below or near the NTV area. Velocity measurements obtained in the BA remained unchanged after use of the monopolar electrode. When aiming the Doppler probe at the ventriculostomy, it was possible to record a faint signal, which corresponded to the movement of the arachnoid surrounding aperture of the NTV. 


\section{DISCUSSION}

There is no doubt that the ability to perform a safe NTV procedure comes with experience. However, the current literature remains vague with regards to the surgical anatomy of the third ventricle and the preferred site on the third ventricle floor where an NTV should be performed.[1-3] At best, the location of a third ventriculostomy is said to be "in front of the mammillary bodies." In our case involving the child, the site for NTV could easily be identified in viro during the procedure, but this is not always the case.[5] Ventriculitis, hemorrhage, or other disease entities can render the floor nontransparent and make identification of a preferred site for NTV difficult. The use of a Doppler probe in those conditions and even in the "easy" patient is a complementary safety measure. The pulsed Doppler probe (FloWire; Cardiometrics, Inc., Mountain View, CA) has an ultrasound frequency of $15 \mathrm{MHz}$, a pulse duration of 0.5 to 2.0 microsec, and a range location from the wire tip of $4.2 \mathrm{~mm}$. The signal is displayed on the screen of the analyzing console (SmartMap; Cardiometrics) and, an acoustic signal is also provided. Both the neuroendoscopic procedure and the visual and acoustic Doppler signals are recorded simultaneously (Fig. 2).

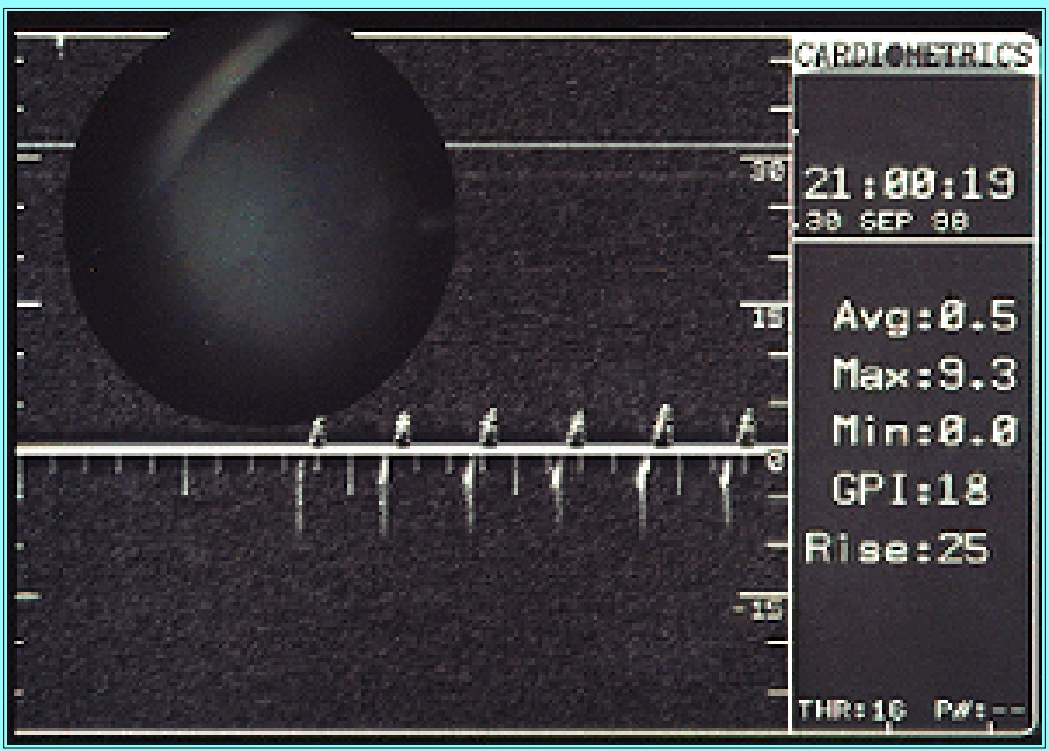

Fig. 2. The top of the figure shows a fold anterior to the chiasm covering the infundibular recess. The probe is at a distance from the floor, and therefore, the velocity of the floor of the third ventricle can be measured. Both the visual and acoustic signals of the floor of the third ventricle resemble that of a cardiac valve. The maximum velocity is $9.3 \mathrm{~cm} / \mathrm{second}$.

Another application of this method for use during neuroendoscopy is the search for blood vessels in nontransparent entities such as cysts, tumors, or during endoscopic deloculation and pellucidotomy. Although the use of circular, lateral gazing ultrasound has been reported, it has been limited to the study of endoscopic anatomical relationships in cadaver specimens.[4] The use of miniature Doppler probes for the location of blood vessels in conjunction with in vivo stereotactic procedures has been reported; however, the probes were not advanced through the endoscope.[6] To our knowledge, we report the first use of a Doppler ultrasound probe passed through a neuroendoscope during an actual procedure.

\section{CONCLUSIONS}

In this paper we describe the use of a Doppler probe, originally built for intracoronary use, that is passed through a flexible neuroendoscope during NTV. The use of the Doppler probe was accurate in locating 
the tip of the BA and aiding in identification of an appropriate site for NTV. The application of this technique in "difficult" NTV procedures in which the floor of the third ventricle is distorted or nontransparent and also in other intraventricular pathology is promising.

\section{References}

1. Buxton N, Vloeberghs M, Punt J: Lilliequist's membrane in minimally invasive endoscopic neurosurgery. Clin Anat 11:187-190, 1998

2. Buxton N, Vloeberghs M, Punt J: Lilliequist's membrane. A re-examination of its importance. Surg Radiol Anat 19 (Suppl):14, 1997 (Abstract)

3. Oka K, Go Y, Kin Y, et al: An observation of the third ventricle under flexible fiberoptic ventriculoscope: normal structure. Surg Neurol 40:273-277, 1993

4. Resch K, Pernecky A: Intraoperative sonography for neuroendoscopic procedures. Minim Invasive Neurosurg 41:122, 1998 (Abstract)

5. Vloeberghs M, Punt J: Neuroendoscopy in children under one year of age. Minim Invasive Neurosurg 41:116, 1998 (Abstract)

6. Yamasaki T, Moritake K, Takaya M, et al: Intraoperative use of Doppler ultrasound and endoscopic monitoring in the stereotactic biopsy of malignant brain tumors. Technical note. J Neurosurg 80:570-574, 1994

Manuscript received December 28, 1998.

Accepted in final form March 5, 1999.

Address reprint requests to: Mr. Michael Vloeberghs, M.D., Queens Medical Centre, Clifton Boulevard, NG72UH, United Kingdom. email: michael.vloeberghs@nottingham.ac.uk. 